\title{
The intensity-velocity phase difference with Magneto-Optical Filters
}

\author{
P. F. Moretti and G. Severino \\ Osservatorio Astronomico di Capodimonte, Via Moiarello 16, 80131 Napoli, Italy \\ Received 25 July 2001 / Accepted 22 November 2001 \begin{abstract}
of the solar atmosphere. The phases are used to quantify the nonadiabatic degree of the acoustic modes and, recently, to infer the characteristics of the source of the solar oscillations. For this reason, the error attributed to the phase obtained from the observations plays an important role in distinguishing between different phenomena. In this paper we discuss the results obtained with the systems using a Magneto-Optical Filter. A model to correct for the contamination induced by the velocity in the intensity signal is described and the phases in the sodium D lines presented. We also discuss the difference between the results obtained when the phase values are computed with a local analysis or when the $\ell-\nu$ diagram is obtained.
\end{abstract} \\ Abstract. The phase difference between the intensity and velocity signals is a powerful diagnostic for the dynamics
}

Key words. Sun: oscillations - instrumentation: miscellaneous - methods: data analysis

\section{Introduction}

The phase difference between the intensity and velocity $(I-V)$ is claimed to be a powerful tool for understanding the nature of the solar oscillations (Deubner et al. 1996; Skartlien \& Rast 2000; Straus et al. 1998; Oliviero et al. 1999). Many attempts have been accomplished to match the results from modelling the $I-V$ values obtained recently from observations. $I-V$ phase differences on the acoustic modes have been used to quantify the nonadiabatic degree of the solar atmosphere (Jimenez et al. 1999). A solar "background" in the $\ell-\nu$ diagram of the phase difference between the intensity and velocity signals, was discovered by Deubner et al. (1990) as a negative phase "plateau" and an inter-ridge regime at high- $\ell$ values and low frequency. This negative phase plateau was confirmed by MDI (Straus et al. 1998) and GONG data (Oliviero et al. 1999).

A better understanding of the dynamics of the solar atmosphere requires multi-wavelength measurements of the $I-V$ phase difference in order to draw a reliable trait of this quantity at different heights.

For this reason, the error associated with the measurements has to be carefully estimated. Solar and instrumental effects make the $I-V$ phase determination uncertain. A phase value is characteristic of a phenomenon and can change accordingly to the physical conditions on the Sun.

Send offprint requests to: P. F. Moretti,

e-mail: moretti@na.astro.it
A measure always performs an average in height, space and time and rarely can a single process be isolated. As a consequence, the error of the phases strongly depends on the phenomena that are mixed in the measurement, and its spread on their statistical properties. Moreover, the calibration and analysis of the data play an important role in identifing the systematic and statistical errors to be attributed to the phase value.

Many systems use narrow passband filters, such as the Magneto-Optical Filter (HiDHN in Mt. Wilson, LOW- $\ell$, Kanzelhöhe, VAMOS, see Rhodes et al. 1988; Tomczyk et al. 1995; Cacciani et al. 1999; Severino et al. 2001, respectively). In this paper we describe a method to estimate the errors and compute the correction in the measured values obtained with these systems. We also discuss the difference between the results obtained when the signal is analysed locally on the solar disk or globally by the spherical harmonics decomposition to get an $\ell-\nu$ diagram.

\section{The intensity and velocity signals obtained through a narrow passband filter}

Typically, a MOF shows a transmission profile with two narrow passbands displaced by some hundreds of milliAngstroms. The two passbands, tuned on the opposite wings (blue, $B$, and red, $R$ ) of the solar line are selected to build dopplergrams as $V_{\text {meas }}=(B-R) /(B+R)$ and intensity images as $I=B+R$ (Cacciani et al. 1988; Cacciani et al. 1999; Cacciani et al. 1997). 
$V_{\text {meas }}$ depends on the slope of the solar line at the two MOF passbands (Parnell \& Beckers 1969) and, as a consequence, changes on the disk accordingly to the motions that displace the wavelength of the line. This slope is also affected by the magnetic field that broadens the line (Ulrich et al. 1993; Marmolino et al. 1997; Cacciani \& Moretti 1997) and by the temperature fluctuations. As a consequence, the signal has to be carefully modelled in order to deduce the true velocity from the measured signal.

In the same way, the intensity obtained through a narrow passband filter depends on the wavelength where it is tuned respect to the solar line profile. The velocity distribution on the solar disk induces spectral shifts by the doppler effect. For this reason, the spatial distribution of a solar intensity image obtained through a narrow passband filter does not depend only on the temperature and height formation (limb darkening), but also on the relative velocity between the observer and the position on the disk.

As a result, the measurements of the velocity and the intensity are mixed with each other. This is what we refer as $I-V$ crosstalk.

How visible is the $I-V$ crosstalk in such systems?

Two cases have to be distinguished, the static and the dynamic ones.

For static we mean the single image (dopplergram or intensity image), where the large velocity offset (corresponding to the rotation plus the Earth-Sun relative velocity and the gravitational redshift, in a range of approximately $\pm 3 \mathrm{~km} \mathrm{~s}^{-1}$ ) and the limb darkening dominate (see Fig. 1). In the intensity image a residual velocity signature can be easily seen: it is due primarily to the solar rotation.

For the dynamic case, we mean when a timeseries of images is obtained. The variation of the signal depends on many effects. First of all it depends on the spatial resolution, e.g. at our resolution of about $4^{\prime \prime}$ in the sodium D lines, the maximum local velocity oscillations are of the order of $300 \mathrm{~m} \mathrm{~s}^{-1}$ while the maximum local intensity oscillations are of the order of $3 \%$. Moreover, on each pixel, that is at any position on the solar disk with its proper relative velocity, 1) the slope of the solar line tuned by the filter and 2) the sampled formation height, both change. This implies a dependence of the amplitude of the oscillations on the solar position. In the intensity signal, the additional effect of the displaced wavelengths induced by the offset velocity is present as in the static case.

In order to make the crosstalk visible in the dynamic case, a local analysis of the oscillations has been performed. Two sets of dopplergrams and intensity images, acquired each minute with a spatial resolution of $4^{\prime \prime} /$ pix, were obtained in 1998 with a sodium MOF at Kanzelhöhe. The two sets of images are displaced in time by $27 \mathrm{~s}$, since the acquisition system was not capable until 2000 of simultaneously providing dopplergrams and intensity images. This effect has been taken into account when computing the phase differences. The images have been calibrated (Moretti \& the MOF Development Group, 2000), geometrically centered and, for each pixel, the time-series

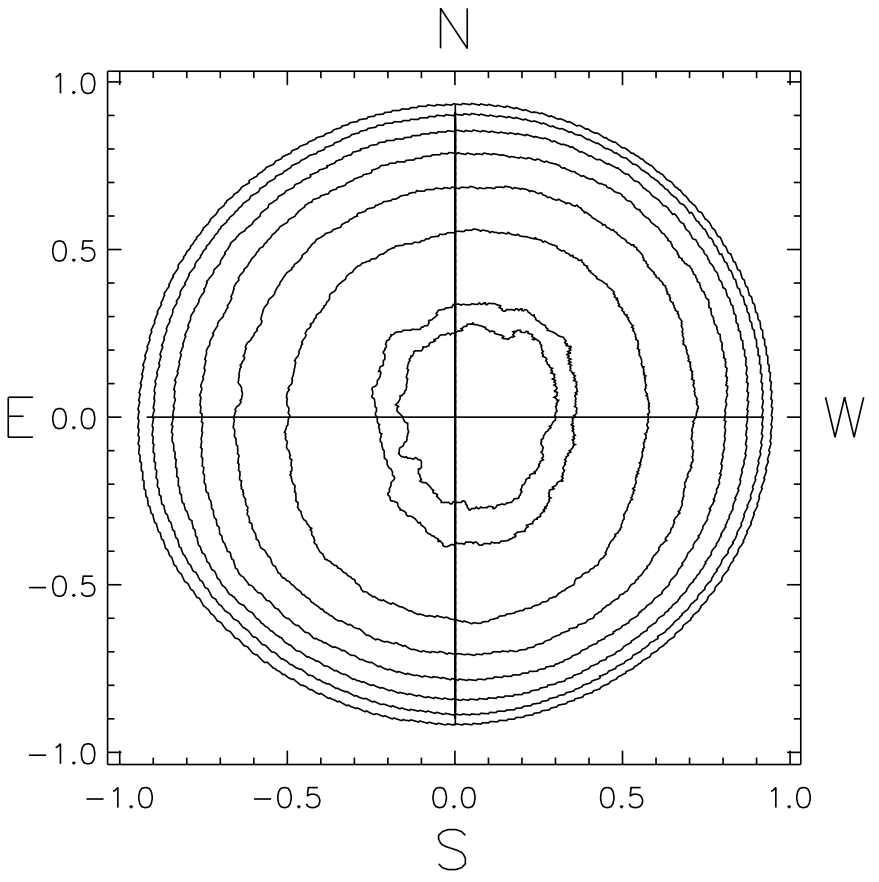

Fig. 1. The iso-intensity curves in a filtergram obtained as the sum over the intensities on the blue and red wings of the sodium D lines with a MOF having a spectral resolution equal to 100000 . The asymmetry in the spatial distribution is due to the velocity contamination.

have been detrended with a polynomial fit and fast Fourier transformed (FFT). Finally, the maps with the spatial distribution of the power at different frequencies have been obtained and normalised for the effect of the detrending filter.

In the local analysis, long time-series would produce a spatial average because of the solar rotation and of the evolution of the structures on the solar surface. In order not to wash out anything but the rotation, the time duration is limited to an observing run of few hours.

The whole data set has been fragmented in a number of sets of a few hours long (we report the case of sets of $256 \mathrm{~min}$ ) in order to limit to a few pixels at disk center the swap due to the rotation.

The power maps have been integrated over the latitude and the longitude respectively to enhance the dependence on the solar rotation (that is in the east-west direction). Since the p-modes are vertical, the power in the five-minute range typically shows a predominant $\sin \theta$ spatial distribution (where $\theta$ is the heliocentric angle). Since the tuning of the filter along the line profile results in scanning the height in the solar atmosphere, an E-W dependence of the oscillation power is also expected because the five-minute power depends on the height in the solar atmosphere. Nevertheless, the intensity power maps show an E-W dependence much stronger than that in the dopplergrams (Figs. 2 and 6a). This additional signal in the intensity is induced by the velocity shift and is in fact mostly visible in the five-minute band, where the velocity power is stronger. 

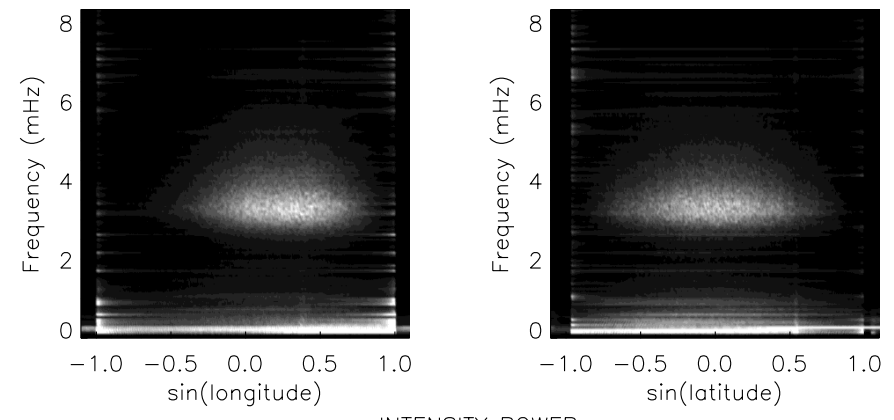

INTENSITY POWER
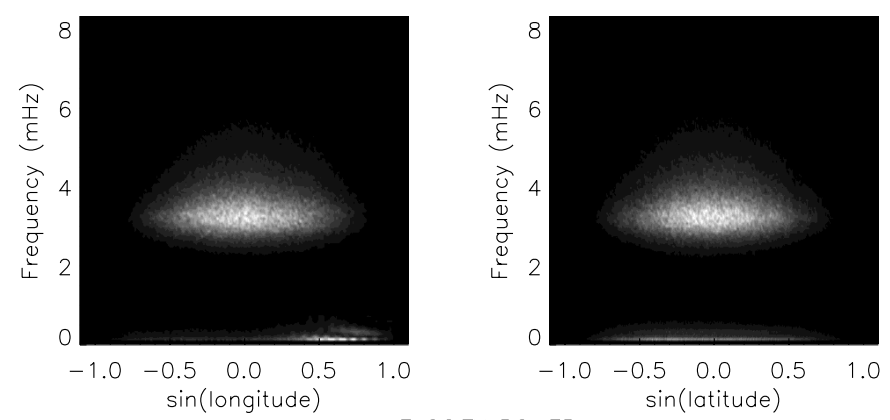

VELOCITY POWER

Fig. 2. The power maps for the intensity (top) and the velocity (bottom) have been integrated over the latitude (left) and the longitude (right) to enhance the dependence on the solar rotation (that is in the east-west direction).

\section{The correction in the intensity for the velocity contamination}

Let the intensity signal $(I=B+R)$ be written as $I=$ $I^{\mathrm{T}, \mathrm{p}}+f(V)$, where the first term is the contribution of the temperature and pressure (hereafter called "true" and indicated as $I^{0}$ ) and the second the one related to the wavelength tuning (that is to the velocity $V$ ).

The calibrated velocity on each pixel is assumed to be a good approximation of the true one, that is $V_{\text {meas }}=V^{0}$. In the linear approximation, a temperature fluctuation induces a slope change whose percentage variations are, equal in the intensity and velocity measurements. At our spatial resolution, local velocity fluctuations of the order of $3 \%$ induced by the intensity are of the same order of the intensity ones induced by the velocity. As a consequence, an additional corrective factor can be included in the definitive phase correction (see next).

In order to estimate the variation of the intensity signal due to the velocity displacements, the slope of the solar line as a function of the velocity offset has to be computed. In fact, in a pure linear approximation of the solar line, a velocity shift would not affect the intensity signal. The spurious intensity signal can instead be largely due to the large slope differences scanned by the two wavelengths sampled by the MOF transmission bands.

The variation of the intensity signal $\Delta I$ can be written as

$\Delta I=\Delta I^{0}+\Delta I^{V}=\Delta I^{0}+\left(\frac{\Delta I}{\Delta \lambda}\right)_{V} \frac{\lambda}{c} \Delta V=\Delta I^{0}+I^{\prime} \Delta V$
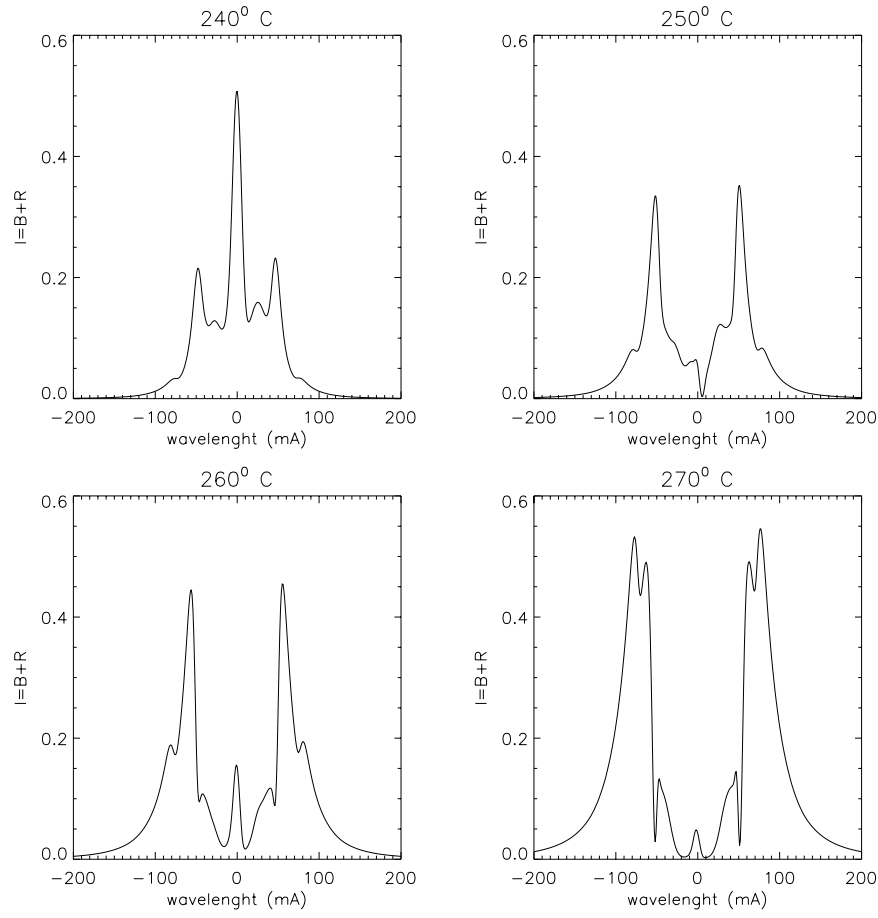

Fig. 3. The sum of the transmission profiles of the two passbands (blue and red) of a sodium MOF at different temperatures of the vapours.
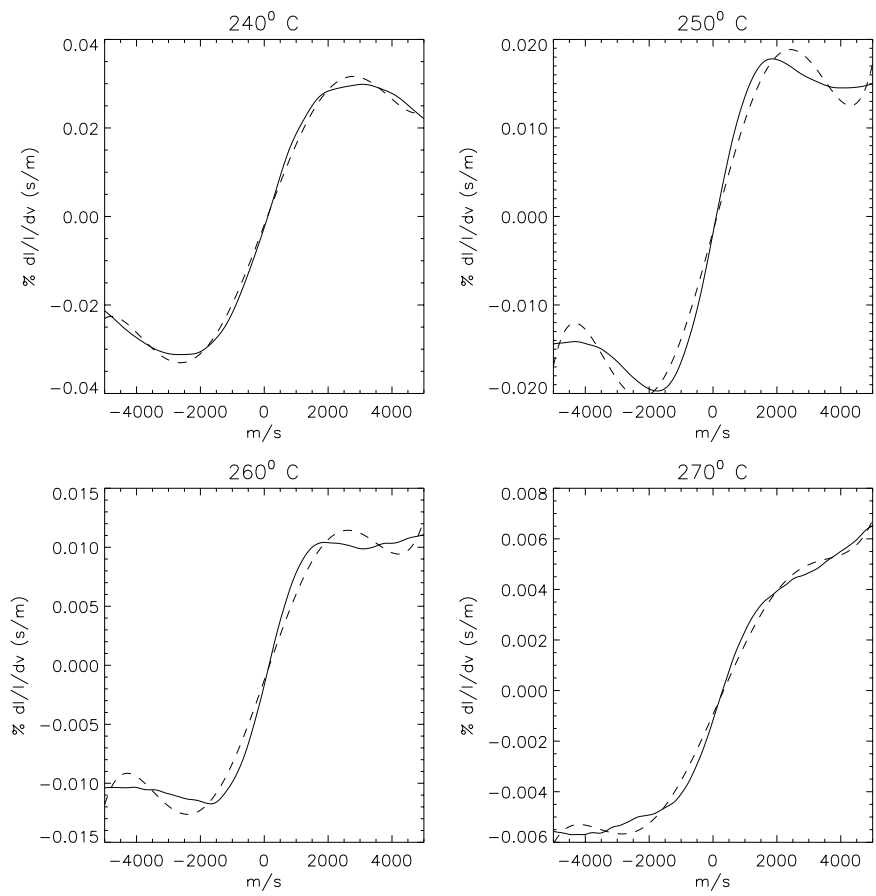

Fig. 4. The percentage of $\frac{\Delta I}{I \Delta V}$ as function of the velocity offset, for MOFs at different temperatures. Fifth order polynomial fits are superimposed (dashed).

where $I^{\prime}$ represents the sensitivity computed at the velocity offset $V$. In a velocity range between -5 and $5 \mathrm{~km} \mathrm{~s}^{-1}$, polynomial fits of different orders have been computed for the $I^{\prime}$ as function of $V$ (for a sodium MOF at different temperatures, Figs. 3 and 4). A 5 th order has been chosen 
Table 1. The fifth order fit coefficients for $\alpha=\frac{I^{\prime}}{I}$ in function of $V$ the at different MOF temperatures (see Fig. 4).

\begin{tabular}{ccccc}
\hline & $235{ }^{\circ} \mathrm{C}$ & $240{ }^{\circ} \mathrm{C}$ & $245{ }^{\circ} \mathrm{C}$ & $250{ }^{\circ} \mathrm{C}$ \\
\hline$A^{0} \times 10^{-5}$ & 2.09 & 1.90 & 1.79 & 1.68 \\
$A^{1} \times 10^{-7}$ & 2.14 & 1.91 & 1.63 & 1.34 \\
$A^{2} \times 10^{-12}$ & 1.86 & 1.91 & 2.02 & 1.85 \\
$A^{3} \times 10^{-14}$ & -1.20 & -1.15 & -1.13 & -1.05 \\
$A^{4} \times 10^{-20}$ & -2.94 & -3.97 & -4.93 & -4.41 \\
$A^{5} \times 10^{-22}$ & 2.16 & 2.29 & 2.58 & 2.62 \\
\hline & & & & \\
$A^{0} \times 10^{-5}$ & 1.48 & 1.26 & 1.08 & 0.94 \\
$A^{1} \times 10^{-7}$ & 1.05 & 0.77 & 0.50 & 0.28 \\
$A^{2} \times 10^{-12}$ & 1.48 & 1.20 & 0.95 & 0.75 \\
$A^{3} \times 10^{-14}$ & -0.81 & -0.55 & -0.35 & -0.15 \\
$A^{4} \times 10^{-20}$ & -3.13 & -2.39 & -1.60 & -0.96 \\
$A^{5} \times 10^{-22}$ & 2.01 & 1.36 & 0.88 & 0.35 \\
\hline
\end{tabular}

to get a good approximation of the fit to the curves at all the temperatures and the coefficients are given in Table 1.

In the range between $\pm 1 \mathrm{~km} \mathrm{~s}^{-1}$, the non-linear terms could be neglected but this choice is too restrictive in the solar case. Between $\pm 3 \mathrm{~km} \mathrm{~s}^{-1}$, the cubic term has to be included, while the other terms contribute less than $10 \%$. Since we deal with percentage intensity variations, we use $\alpha=\frac{I^{\prime}}{I}$ and for the power, it can be written

$$
\left|\frac{\Delta I}{I}\right|^{2}=\left|\frac{\Delta I^{0}}{I}\right|^{2}+\alpha^{2}|\Delta V|^{2}+2 \alpha \frac{\Delta I^{0}}{I} \Delta V \cos \left(\Delta I^{0} \Delta V\right)
$$

where the first term is related to the temperature fluctuations, the second to the wavelength tuning and the last to the phase relation in time between the intensity and velocity signals, that is their phase difference $\phi_{I-V}$. In order to remove the contamination due to the velocity, at any point on the solar disk and any frequency, the Eq. (1) is solved for $\frac{\Delta I^{0}}{I}$ as:

$$
\begin{aligned}
\frac{\Delta I^{0}}{I}= & -\alpha \Delta V \cos \left(\Delta I^{0} \Delta V\right) \\
& \pm \sqrt{\alpha^{2}|\Delta V|^{2}\left(\cos ^{2}\left(\Delta I^{0} \Delta V\right)-1\right)+\left|\frac{\Delta I}{I}\right|^{2}} .
\end{aligned}
$$

One of the two solutions of (2) is chosen. It requires $\Delta V$, $\frac{\Delta I}{I}, \alpha$ and $\cos \left(\phi_{I-V}\right)$ to be known. The first two are deduced from the measured powers, $\alpha$ is computed using the real velocity distributions and the transmission profiles (see Fig. 5). This parameter is in some sense a little bit uncertain as the real transmission profiles are not always perfectly known (the real working temperature of the vapours of the MOF can be different from the one measured on the glass cell containing the vapours); anyway,
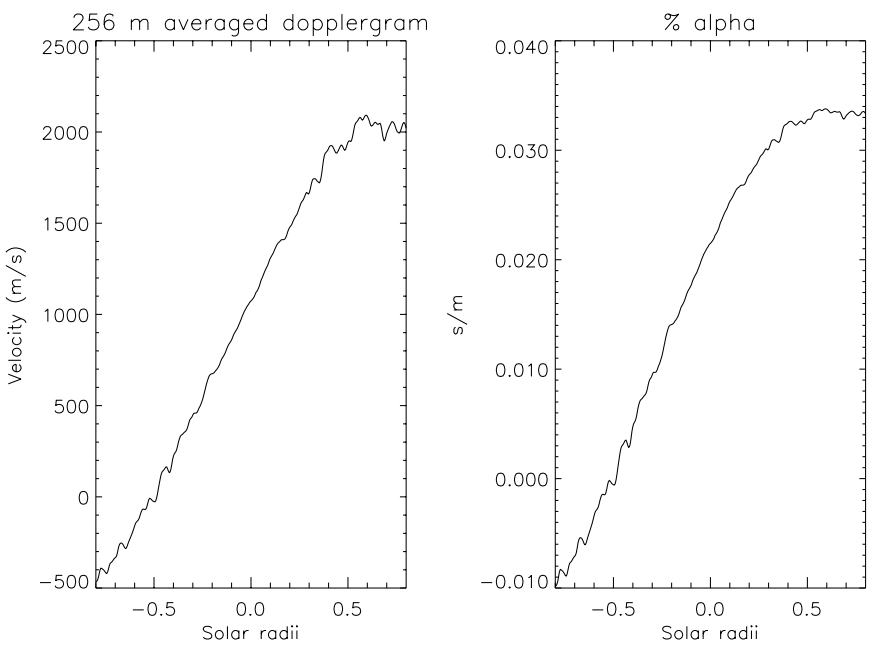

Fig. 5. Left: the scan along the equator of a 256 min average dopplergram. Right: the intensity signal induced by a velocity shift of the line has been computed using the averaged doplergram (a theoretical mean velocity spatial distribution could also be used). The scan along the equator of the percentage of $\alpha$ for a MOF at $240^{\circ}$ is shown in function of the velocity offset. The induced $\left(\frac{\Delta I}{I}\right)$ can be estimated from this curve introducing the velocity displacement at the position on the disk relative to the velocity offset.

this parameter can not be varied dramatically, as it affects the positivity of the factor in the square root in Eq. (2).

The $\cos \phi_{I-V}$ is the core parameter. It is now free to vary and is related to the phase difference as it will be inferred from the cross-power between the measured intensity and velocity signals and that we want to correct for the crosstalk (for this reason, we will continue to write this parameter $\cos \phi_{I-V}$ to distinguish it from the $\phi_{I-V}$ deduced by the cross-power, see Sect. 4). The choice of this parameter is constrained by the dependence of the solution of Eq. (2) along the E-W direction: we want the intensity spatial distribution on the disk to be similar to the velocity one (Fig. 2). The ratio between the slopes of the average E-W and N-S power distributions has been chosen to control the goodness of the correction (Fig. 6a). A sample of the corrected power map is shown in Fig. 6b. The value of $\cos \phi_{I-V}$ depends on the frequency and the values are mainly two: around 0.3 and 1.0 , corresponding respectively to the five-minute band and elsewhere. The sign of $\cos \phi_{I-V}$ is not unique, as the other solution of (2) with $-\cos \phi_{I-V}$ can be chosen as well. Its value in the five-minute band varies from 0.2 to 0.4 and, since $\cos \phi_{I-V}=\cos \left(180-\phi_{I-V}\right)$, the corresponding possible $\phi_{I-V}$ spans from $+60^{\circ}$ to $+120^{\circ}$.

\section{The crosstalk in the $I-V$ phase difference}

How does the crosstalk described above affect the $I-V$ phase difference results?

The phase difference between the variation of the measured intensity and velocity signals $\left(\phi_{I-V}\right)$ is evaluated via 

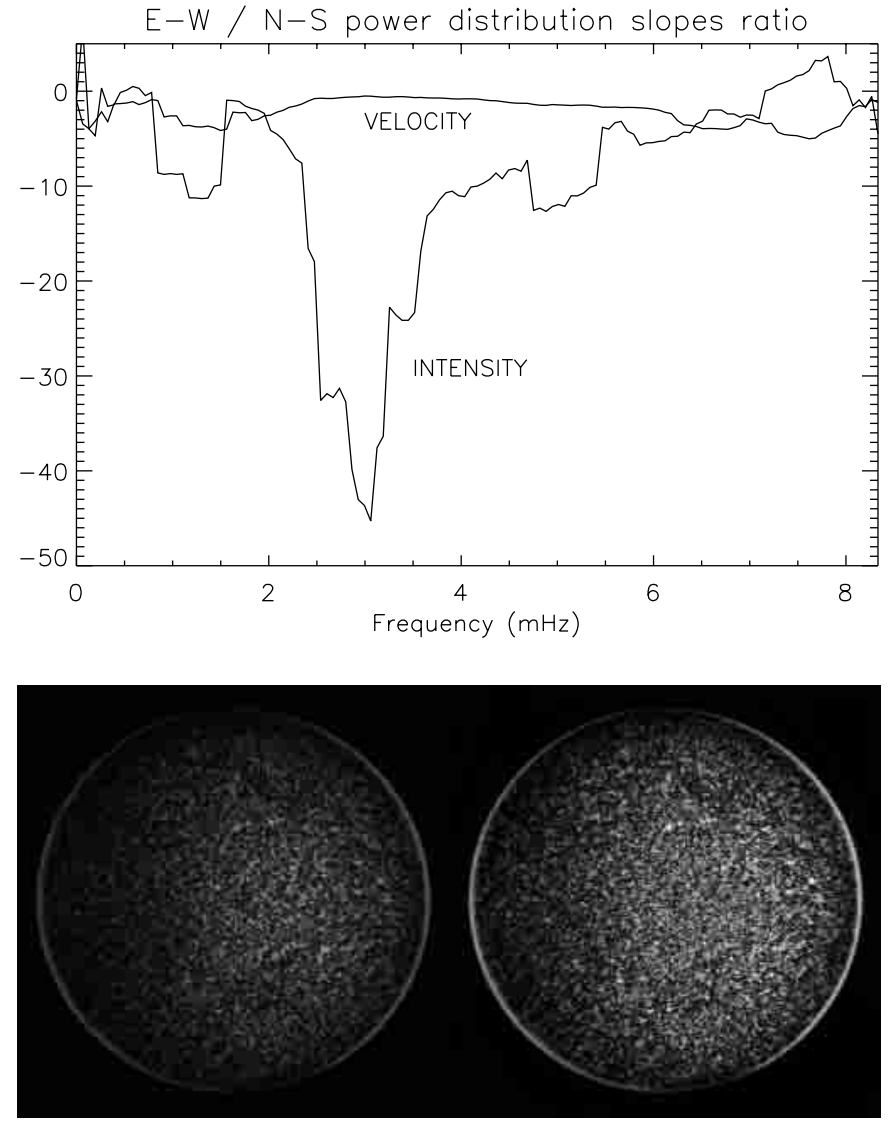

Fig. 6. Top: the correction in the power maps has been constrained by the ratio between the slopes of the E-W and N-S spatial distributions. The frequency dependence is shown before the correction. Bottom left: the map of the measured $\frac{\Delta I}{I}$ at $3.58 \mathrm{mHz}$. Right: the $\frac{\Delta I^{0}}{I}$ obtained, at the same frequency, from Eq. (2) with $\cos \phi_{I-V}=0.4$. The black-to-white scale goes from 0 to $0.2 \%$.

the crosspower as:

$$
\begin{aligned}
\phi_{I-V} & =a \tan \left(\frac{\operatorname{Im}\left(\frac{\widetilde{\Delta I}}{I} \widetilde{\Delta V}^{*}\right)}{\operatorname{Re}\left(\widetilde{\frac{\Delta I}{I}} \widetilde{\Delta V}^{*}\right)}\right) \\
& =a \tan \left(\frac{\operatorname{Im}\left(\widetilde{\frac{\Delta I^{0}}{I}} \widetilde{\Delta V}^{*}\right)}{\operatorname{Re}\left(\widetilde{\frac{\Delta I^{0}}{I}} \widetilde{\Delta V}^{*}+\alpha\left|\widetilde{\Delta V}^{*}\right|^{2}\right)}\right)
\end{aligned}
$$

where the superscripted star indicates the complex conjugate and the tilde refers to the FFTs of the related quantities.

We distinguish two cases: the local analysis and the spherical harmonics decomposition.

\subsection{The local analysis}

In the local analysis, each point on the disk is treated with its own velocity offset and velocity power. The error in the $I-V$ phase difference depends on the relative strength between the instrumental crosstalk contribution and the intensity oscillations due to the temperature fluctuations,

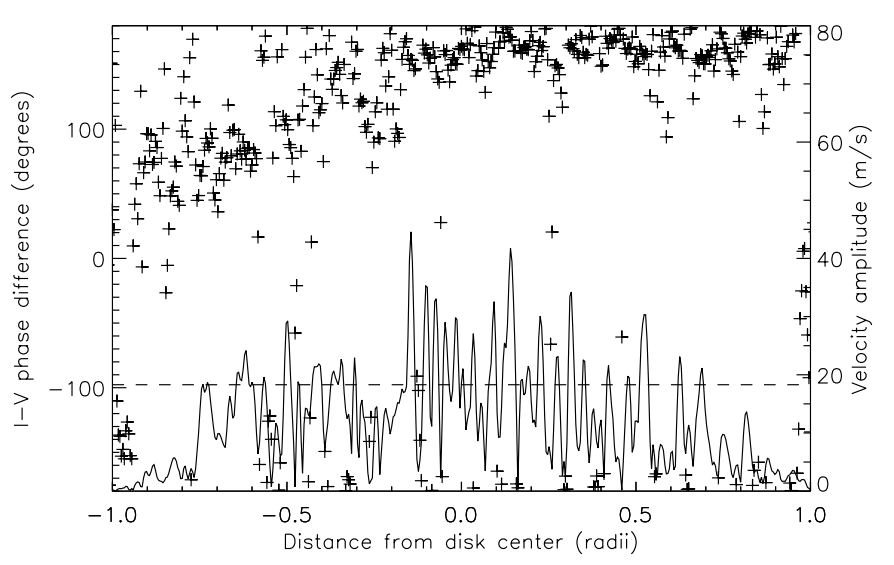

Fig. 7. Scans along the equator for the maps at $3.32 \mathrm{mHz}$. The $I-V$ phase difference (crosses) before the crosstalk correction and the velocity amplitude (solid line). The dashed line refers to the threshold in amplitude used to select the peaks in the oscillatory power.

hereafter denoted as $\gamma=\frac{\alpha \Delta V}{\frac{\Delta I^{0}}{I}}$. We remark that the error in the phase difference determination is attributed only to the influence of the velocity displacements on the intensity signal (we neglect for the moment the influence of the intensity on the velocity). Any pure instrumental crosstalk acts at zero phase: any displacement in $V$ immediately causes an instrumental intensity fluctuation. In Fig. 8 a vector representation of the crosstalk effect is sketched. The intensity induced by a velocity displacement is always parallel to the velocity and rotates the total intensity vector. The sign of the contamination depends on the convention adopted for the intensity and velocity fluctuations; when the velocity is assumed positive for a redshift, the crosstalk makes the phase become closer to zero, while to $180^{\circ}$ for a positive blueshift velocity. A spurious velocity signal induced by an intensity fluctuation (interpreted as a slope change due to temperature variations) behaves the same way.

The contamination strongly depends on the different phenomena resolved on the solar disk. The ratio between the velocity and intensity fluctuations depends on the position on disk (due to projective factors, and on their dependence on the height of the atmosphere, that is on the line wavelength, i.e. on the offset velocity).

In the procedure we use, the real distributions are taken into account since the real data are used to compute $\gamma$ itself. Unfortunately, the true $\phi_{I^{0}-V}$ can not be explicitly expressed in terms of $\gamma$. Nevertheless, some limit cases are distinguished. If $\phi_{I^{0}-V} \simeq 0^{\circ}$ or $180^{\circ}$, that is almost parallel, the error is not large since the measured one tends to be parallel too. If $\phi_{I^{0}-V} \simeq 90^{\circ}$, the error depends on $\gamma$. When $\gamma \ll 1$, the measured $\phi_{I-V}$ is plotted in Fig. 9 . In our data (with a MOF at $\simeq 270{ }^{\circ} \mathrm{C}$ ), $\gamma=\simeq 0.1$.

The measured $\phi_{I-V}$ depends, as expected, on the position on the disk because of both crosstalk and different sampled heights in the solar atmosphere (see Fig. 7). A rough correction based in Fig. 9 suggests the true phase averaged on the whole disk to be lower by $\simeq 30^{\circ}$, but the 


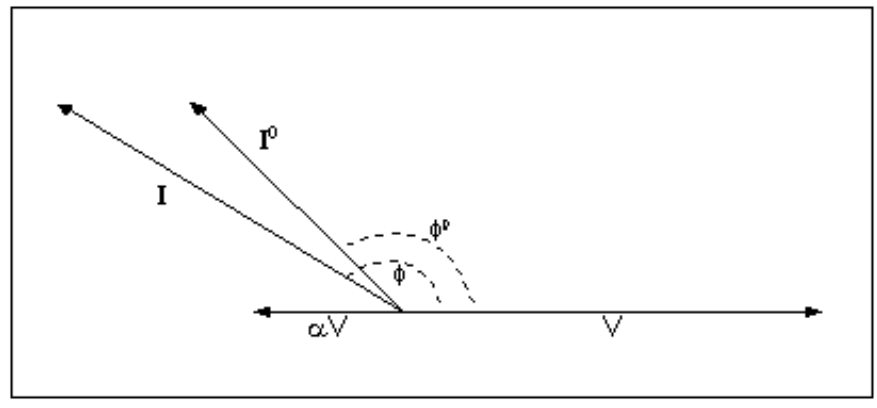

Fig. 8. A vector representation of the $I-V$ phase difference in presence of crosstalk. The intensity induced by a velocity displacement $\alpha V$ is always parallel to the velocity and rotates the total intensity vector. The sign of the contamination depends on the convention adopted for the intensity and velocity fluctuations, and makes the measured intensity vector $(I)$ to be parallel. A spurious velocity signal induced by an intensity fluctuation (interpreted as a slope change due to temperature variations) behaves the same way.

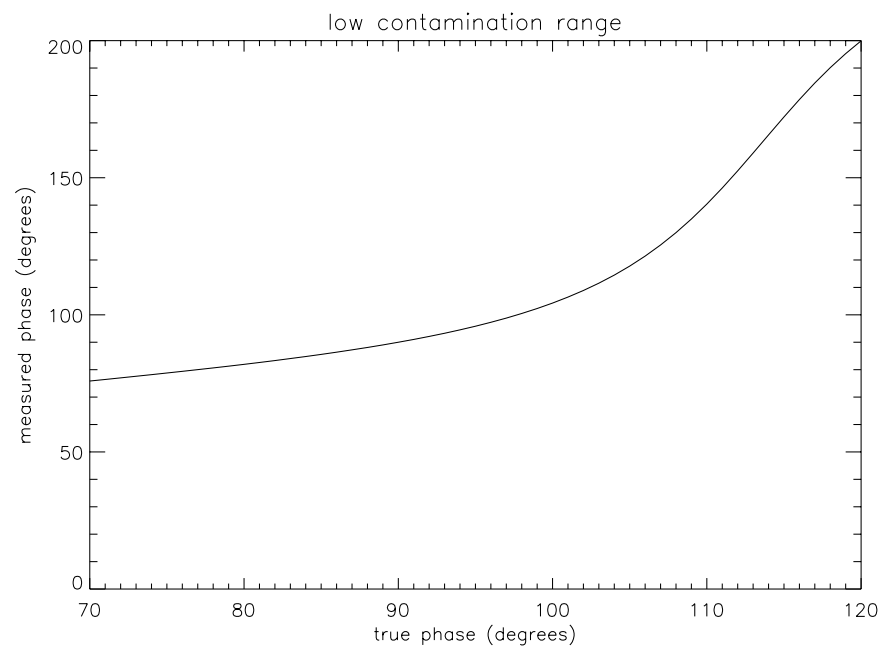

Fig. 9. The measured $\phi_{I-V}$ as function of the true $\phi_{I^{0}-V}$ in the case of low crosstalk contamination and $\phi_{I^{0}-V}=\simeq 90^{\circ}$.

limit case can not always be used as we also obtain measured phases far from $90^{\circ}$.

This approach is based on the computation of $\gamma$ as inferred by the local velocity amplitude estimated from the power maps. That is, it will be corrected where the power is large (typically, the constructive interference patterns of the five-minute oscillations). Nevertheless, if a phenomenon does not show a significant power in the velocity signal, the resulting measured phase could be contaminated as well. This is the case, for example, of single high velocity pulses, randomly distributed in time but with a definite phase relation with the intensity fluctuations: the velocity power could be low, but the velocity displacement of the pulse largely modifies the intensity signal, leading to a contaminated phase. A scenario with fast downflows at minute timescales is invoked to justify the trait of the background in the $\ell-\nu$ diagram of the $I-V$ phase difference (Skartlien \& Rast 2000).
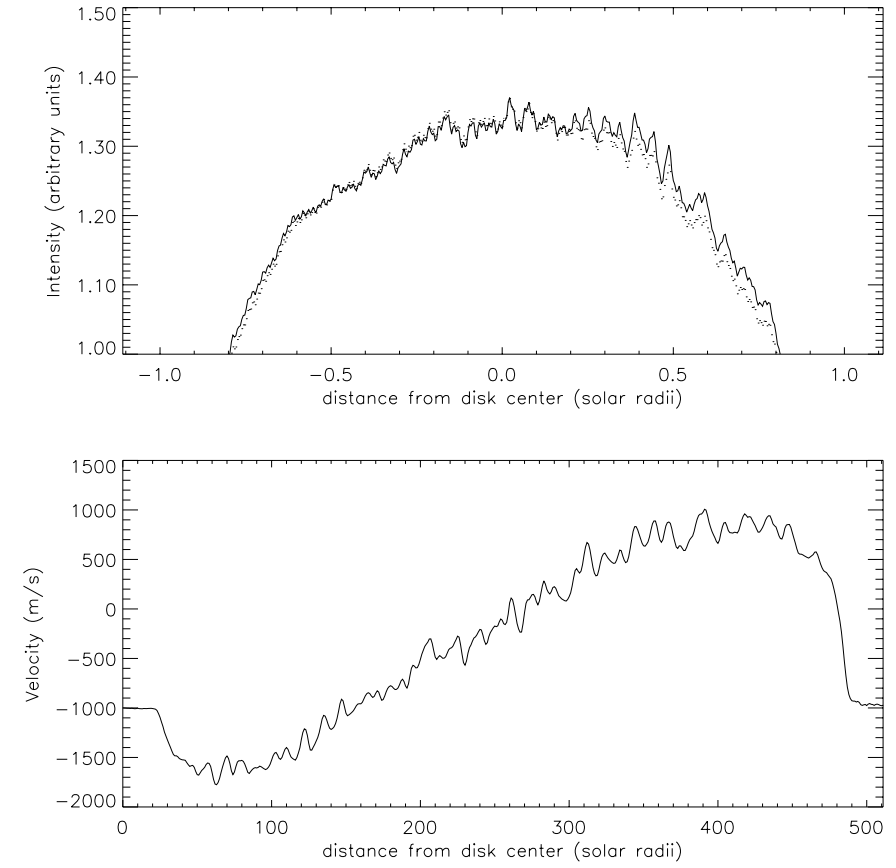

Fig. 10. Top: a scan along the equator for an intensity image before (solid) and after (dashed) the correction (sodium $\mathrm{MOF}$ at $270{ }^{\circ} \mathrm{C}$ ). Bottom: the scan along the equator for the simultaneous dopplegram.

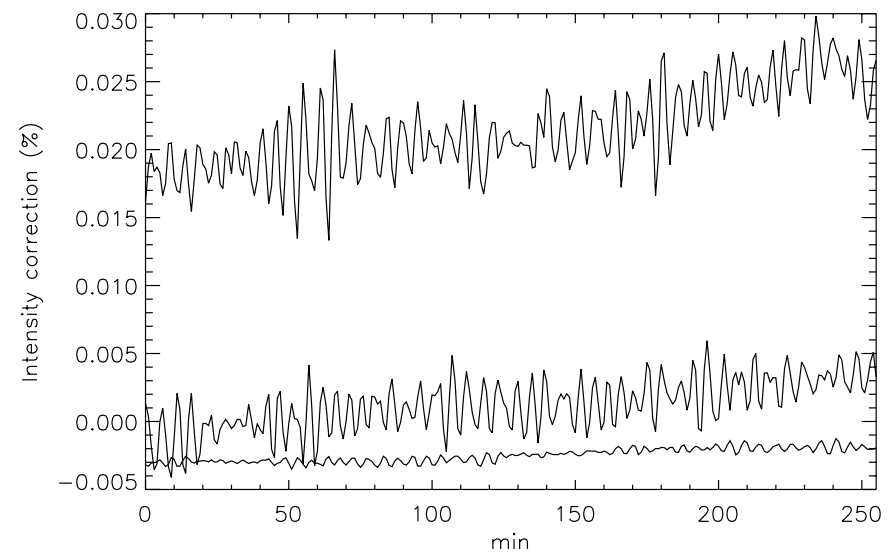

Fig. 11. The correction in the intensity images is proportional to the velocity offset as it tunes the filters at higher slopes of the solar line. From top to bottom, the timeseries of the corrections for the intensity are shown for three samples on the equator at $33{ }^{\circ} \mathrm{W}$, center disk and $33^{\circ} \mathrm{E}$ respectively.

\subsection{The "safe" correction}

Since the crosstalk is primarily due to the different tuning of the filter transmission profiles, the correct procedure to compute the $I-V$ phase difference is to restore the intensity images as they would have been acquired at a common wavelength. In practice, the $I^{0}=I-\alpha V$ has to be computed for each position on the solar disk (in our case, the velocity signal has also been interpolated to the acquisition time of the intensity image). In Figs. 10 and 11 the results of such a restoration are shown for a 

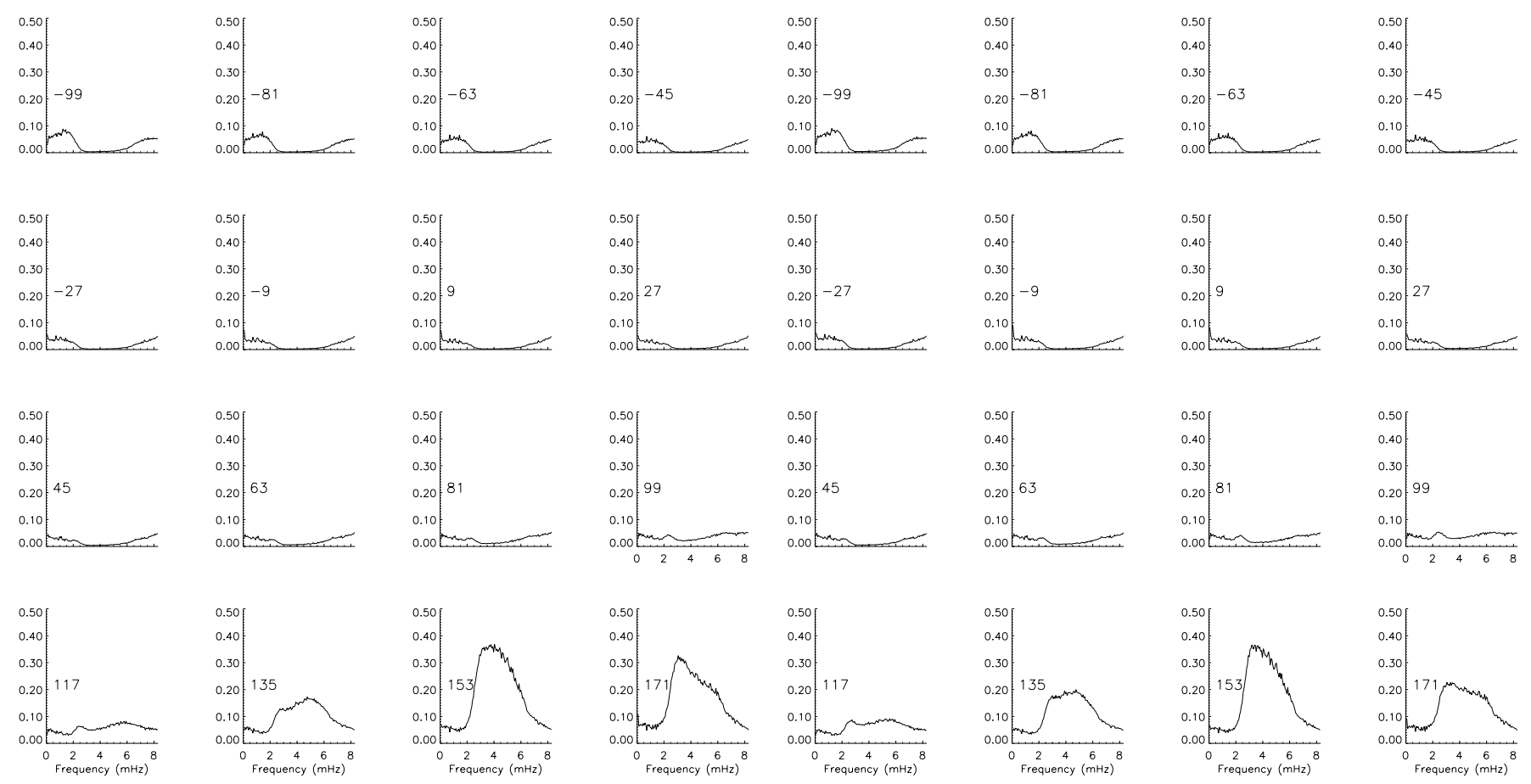

Fig. 12. For each $I-V$ phase difference map, the $360^{\circ}$ range has been divided into 20 intervals, $18^{\circ}$ wide each. At any frequency, a value equal one has been assigned to those pixels showing the phase value corresponding to an interval, zero elsewhere. The number of these pixels inside the solar disk out to 0.5 radii, divided by the total number of pixels inside the same area, gives the "coverage" of that phase at the selected frequency in the part of the disk where the $p$-modes dominate (since their motions are vertical). Before (left) and after (right) the $I-V$ crosstalk correction for a sodium MOF at $270{ }^{\circ} \mathrm{C}$. The expected value for an uniform distribution is 0.05 .

$\mathrm{MOF}$ at $270{ }^{\circ} \mathrm{C}$. The correction now acts independently on the velocity fluctuations and removes the residual velocity offset from the raw intensity images.

The analysis has been repeated using the corrected intensity images.

The $360^{\circ}$ range has been divided into twenty intervals, $18^{\circ}$ wide each. At any frequency, a weight equal to one has been assigned to those pixels on the disk showing the phase value corresponding to that interval, zero elsewhere. The number of these pixels inside the solar disk out to 0.5 radii, divided by the total number of pixels inside the same area (hereafter called "the half radius area"), gives the "coverage" of that phase value at the selected frequency, that is its probability to be found in the part of the disk where the p-modes interference pattern dominates (because their motions are practically vertical). The $0.5 R$ area has been chosen to avoid to introduce projective factors (based on the properties of the different phenomena). Figure 12 shows the frequency distributions of the probabilities to find a phase for each selected interval, for the raw and corrected images: the interval corresponding to the value of $+153^{\circ} \pm 18^{\circ}$ is the most populated in the five-minute band, and after the correction, the $+171^{\circ}$ interval is depleted. The presence of different populations of the phase values has been demonstrated in the $\ell-\nu$ diagram (Straus et al. 1998; Oliviero et al. 1999) and using the local analysis as well (Moretti et al. 2001b): the positive component and another one, at $\simeq-140^{\circ}$. We attribute this latter value to the "solar background" at the sodium D lines formation layer.

In order to handle the error of the phase, the values for the modes have been selected as those corresponding to the locations where the velocity power is greater than a threshold (the higher is the threshold, the larger is the probability to isolate the phase of the modes in the five-minute band, see Fig. 1 of Moretti et al. 2001a). The threshold has been chosen as twice the mean velocity amplitude computed from the power maps: it changes approximately from $1 \mathrm{~m} \mathrm{~s}^{-1}$ at the low frequencies to $15 \mathrm{~m} \mathrm{~s}^{-1}$ in the five-minute band. The values of the phase at these locations have been successively averaged over the solar disk and their frequency dependence is shown in Fig. 14.

The phase value corresponding to high velocity power locations, averaged between 2.9 and $3.6 \mathrm{mHz}$ in the inner half radius area, is lowered from $+150^{\circ} \pm 11^{\circ}$ to $+145^{\circ} \pm 11^{\circ}$ after the correction. If the threshold to isolate the modes is halved, the phase is lowered, both in the cases before and after the correction $\left(+133^{\circ} \pm 40^{\circ}\right.$ and $+128^{\circ} \pm 20^{\circ}$ respectively), because the phases are mixed to other phenomena. Nevertheless, the systematic error is always of about $5^{\circ}$ and the spread of the phase values is reduced. The high velocity power locations in the five-minute band are usually considered the place where the interference of the oscillations acts constructively, and for this reason we attribute these phase values to the "modes" also in the local analysis. Other phase values seem to be randomly 

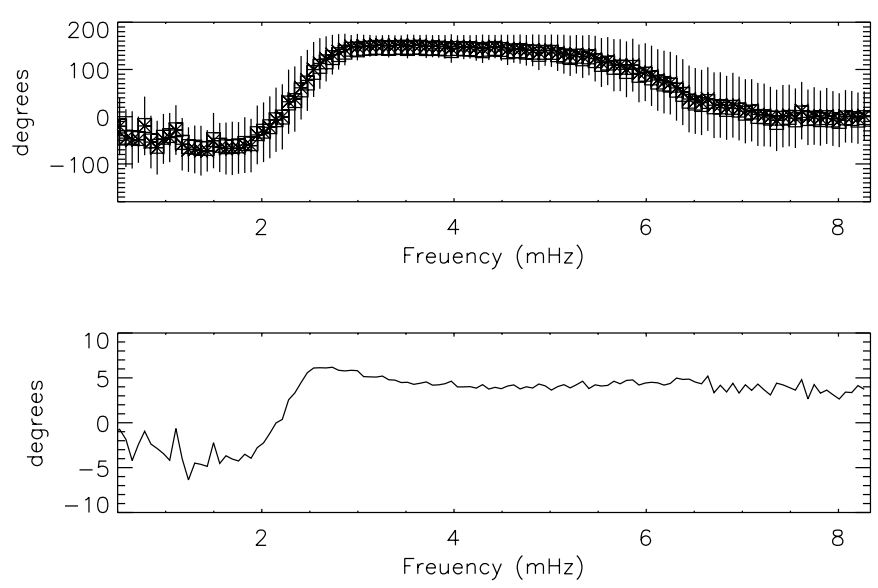

Fig. 13. Top: from the local analysis of $4^{\prime \prime} /$ pix images: the $I-V$ phase differences (before, crosses, and after the crosstalk correction, squares) corresponding to the high velocity power locations (that is the $p$-modes at the five-minute band) have been averaged over the disk. The error bars refer to the values corrected for the crosstalk contamination. Bottom: the difference between the values before and after the correction.

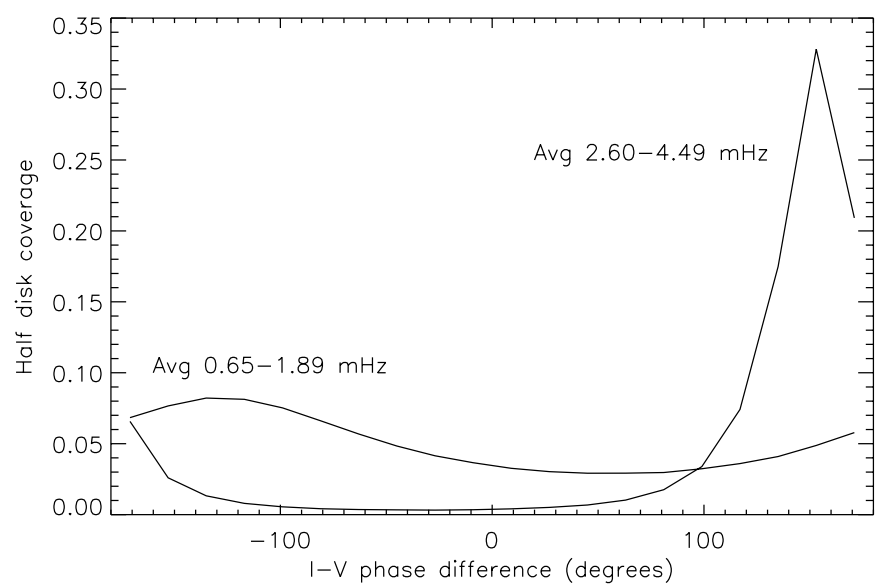

Fig. 14. The probabilities in finding a $I-V$ phase value have been averaged in the low frequency and five-minute bands. Two populations are visible in the local analysis as well as in the $\ell-\nu$ diagram. We associate the population with the positive value to the $p$-modes, while the negative to the solar background. The expected value for an uniform distribution is 0.05 .

distributed on the disk, but at disk center in the fiveminute band, the $-140^{\circ}$ value occurs where the velocity power is low, while it dominates at low frequencies (Moretti et al. 2001b; Oliviero et al. 1998).

In order to enhance the presence of this "background" population, an average of the probabilities in the low frequency range (between 0.65 and $1.89 \mathrm{mHz}$ ) has been performed as shown in Fig. 14. In this case, the evaluation of the "background" values have been estimated through a Gaussian fit of the probability curves in Fig. 15, and led to $-138^{\circ} \pm 50^{\circ}$ and $-127^{\circ} \pm 55^{\circ}$ for the data before and after the correction respectively. This means that, even if the background does not show an oscillatory power comparable to the modes at any frequency, the ratio between the velocity and intensity fluctuations associated with the

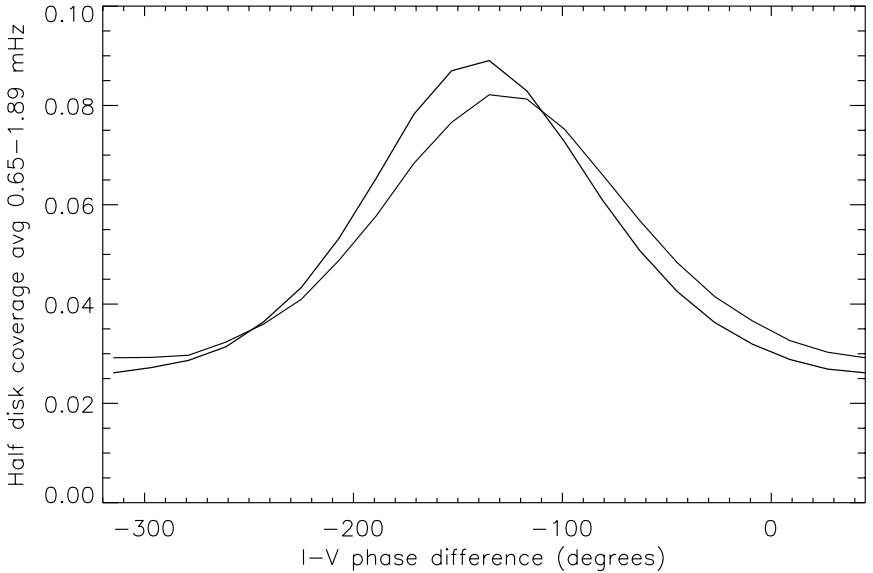

Fig. 15. The probability in finding a $I-V$ phase difference value on the solar disk have been averaged over the frequency for the low frequency band $(0.65-1.89 \mathrm{mHz})$ before (thick) and after the correction. This is the distribution of the "solar background". The expected value for an uniform distribution is 0.05 .

phenomenon underlaying the background is comparable to that of the modes at $4^{\prime \prime}$ spatial resolution. In summary, the correction procedure for the crosstalk contamination in the sole intensity images for a sodium MOF at $270{ }^{\circ} \mathrm{C}$ (described in 4.2), has lead to lower values in the modulus of the $I-V$ phase difference of approximately 5 degrees in the modes. This error depends, as previously described, on the instrumental parameters.

The phase value we obtained using the local analysis is $145^{\circ} \pm 11^{\circ}$ for the $p$-modes averaged over the disk, that is, over the formation layers of the sodium $D$ lines (approximately between 550 and $950 \mathrm{~km}$ ).

Another procedure to infer the phase values independently of the instrumental characteristics can be used: it is based on the selection of the values in the region of the disk where the contamination is negligible. In fact, where the relative velocity is approximately zero during the observation run, the intensity variations induced by velocity shifts are balanced between the opposite blue and the red wings of the solar line. Accordingly to Fig. 5 and for a MOF at $270{ }^{\circ} \mathrm{C}$, the region of the disk where $\alpha<0.5 \times 10^{-5}$ out to $0.5 R$ has been selected: this is the part where $\gamma$ is always smaller than $10^{-3}$ at all frequencies. The phase in correspondence of the powerful locations has been selected and the result is shown in Fig. 16: the average value between 2.9 and $3.6 \mathrm{mHz}$ is now $+100^{\circ} \pm 40^{\circ}$, and refers to solar heights between approximately 700 and $900 \mathrm{~km}$.

\section{The $\ell-\nu$ diagram}

When the spherical harmonic decomposition is applied to the data, the results are typically shown through the $\ell-\nu$ diagram. The power and phase, corrected for the rotational splittings, $\ell-\nu$ diagrams have been obtained for the sets of dopplergrams and intensity images previously described. Since the two sets were not simultaneous, the correction to the phase has been introduced as well 

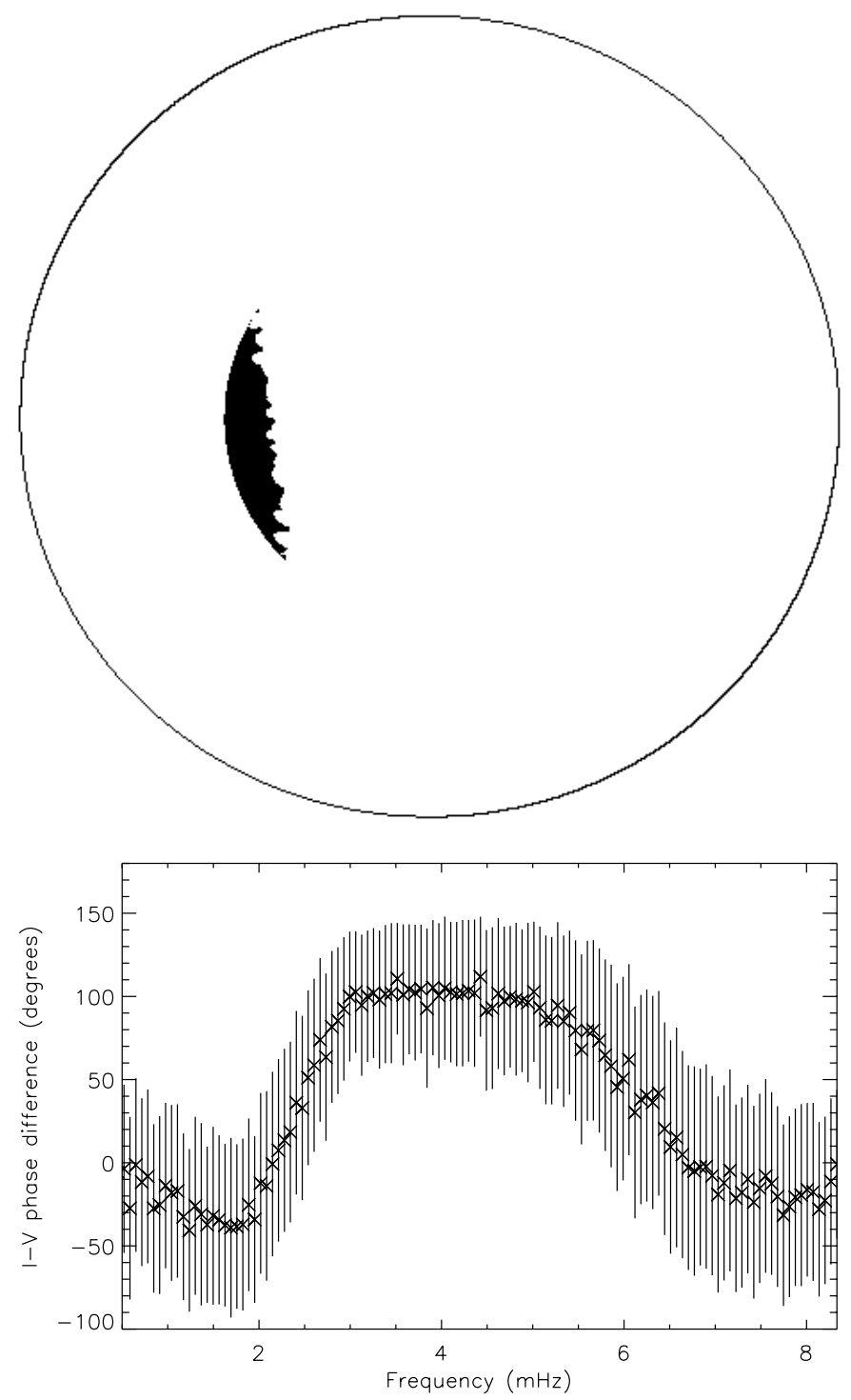

Fig. 16. The $I-V$ phase differences (bottom) corresponding to the high power locations have been selected in that area of the disk where the contamination is negligible (top) and averaged over the disk. In that area, the wavelengths tuned by the filter select a particular formation layer (see also text).

as in the phase maps in the local analysis (see Fig. 2 of Moretti et al. 2001a). The obtained phase values practically confirm the results obtained by Oliviero et al. (1998) in the sodium D lines with an analogous MOF-based system installed in Naples (Moretti et al. 1997). Two main regions, independent of $\ell$, are visible in the frequency domain: $\mathrm{a}+155^{\circ}$ component corresponding to the modes at $\nu>2.5 \mathrm{mHz}$ and $-140^{\circ}$ elsewhere. The correction for the $I-V$ crosstalk has been performed using the corrected intensity images previously described (see Sect. 4.2). The results are shown in Fig. 17.

Even if the data do not show reliable results for the very low $\ell$, we remark that the lowest $\ell$ are the more affected by the crosstalk. This is due to the topology of the contamination over the disk, that primarily draws the rotation (see Fig. 5). At any frequency, the spherical

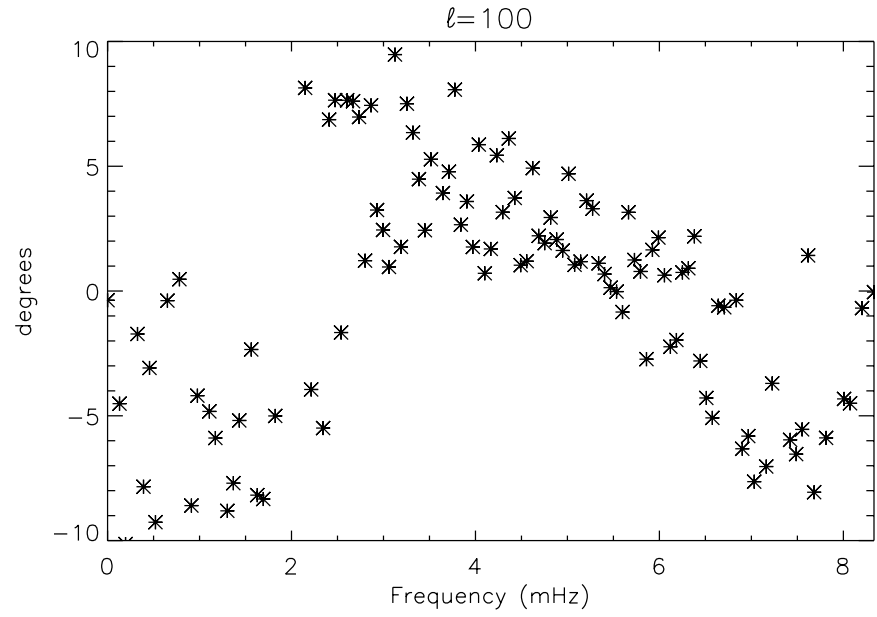

Fig. 17. The difference between the $I-V$ phase values at $\ell=100$ before and after the crosstalk correction.
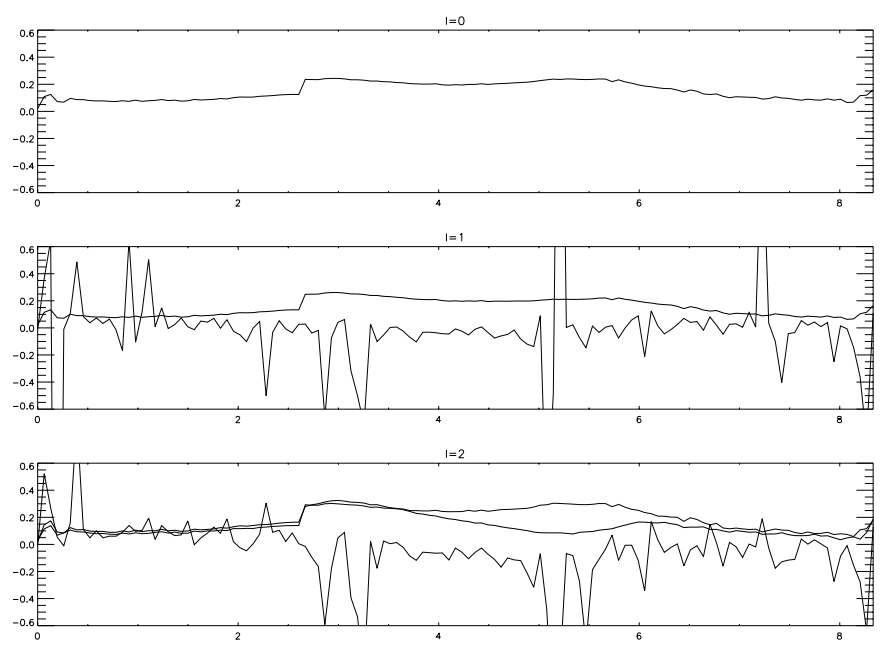

Fig. 18. At any frequency, the spherical harmonic mask corresponding to the different $\ell, m$ coefficients have been applied to the maps of $\Delta V$ and $\frac{\Delta I^{0}}{I}$ and the corresponding $\gamma_{\ell, m, \nu}$ have been obtained. On $y$-axis the frequency is reported. From top to bottom $\gamma_{\ell, m, \nu}$ for $\ell=0,1,2$ respectively. On each panel the $\mathrm{m}$ curves are shown ( $m=0$ is always the lower, $m=\ell$ the larger). The strong $m$ dependence at low $\ell$ is due to the morphology of the velocity spatial distribution. In the $m=0$ curves many spikes are present due to the divergence of $\gamma$ when the power is low.

harmonic masks corresponding to the different $\ell, m$ coefficients have been applied to the maps of $\Delta V$ and $\frac{\Delta I^{0}}{I}$ and the corresponding $\gamma_{\ell, m, \nu}$ have been obtained, where the underscript $\ell, m$ refers to the adopted mask and not to the single mode. The $\gamma_{\ell, m, \nu}$ do not represent the correction to introduce in the results but clearly show the strong $m$ dependence of the contamination (Fig. 18). The corrected phase values on the peaks are no longer approximately independent on the frequency, but increase with it. This is independent on $\ell$ for those values where the data show an acceptable $S / N(10<\ell<350)$. It is clear that, according solely to the relative powers between the five and three-minute ranges, the correction in the three-minute 


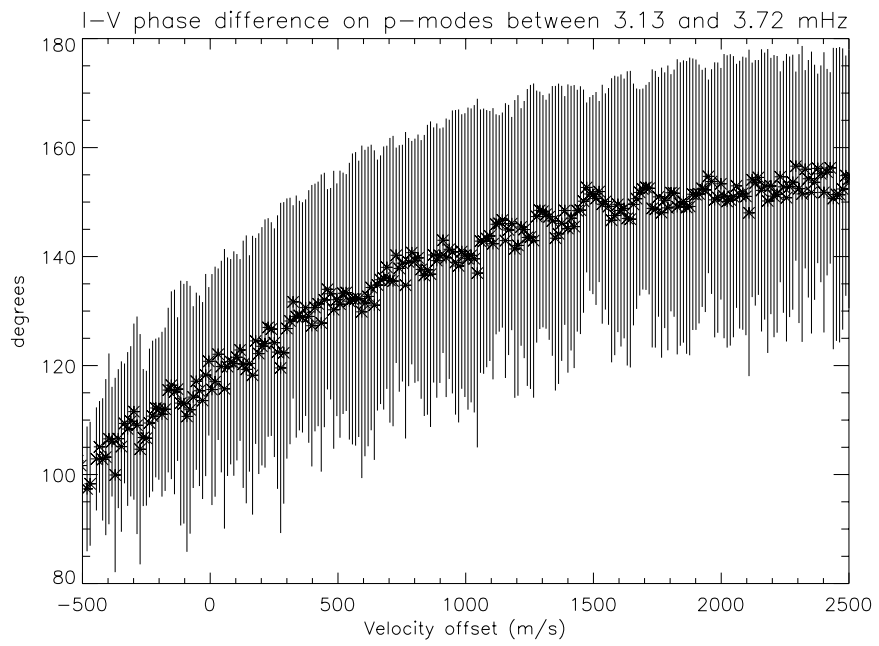

Fig. 19. For any phase map, the phases corresponding to the high velocity power locations (associated with the $p$-modes) have been selected according to the relative velocity offset (positive for reddening). The average between 3.13 and $3.72 \mathrm{mHz}$ is shown. The error bars have been obtained as the average of the spreads over both the disk and the frequencies.

band cannot make the values lower than in the five-minute band. But, the phase difference shows a discrepancy between the local and global analysis in the three-minute band: the phase is larger than the five-minute values in the $\ell-\nu$ diagram and lower in the local analysis. This result can be attributed to a different dilution of the phenomena when they are averaged in space. In fact, since the velocity amplitude in the three-minute band is small, a selection of the modes using the local analysis can not be performed as clearly as in the five-minute band. As a result, the phase values of the p-modes phase are lowered by the mixing with the other phenomena.

\section{The dependence with height of the $p$-modes phases}

In Fig. 19 we show the $I-V$ phases for the locations where the five-minute velocity power is high (that is where the $p$-modes interfere constructively), using the velocity offset as a spatial coordinate. We have then computed the formation heights at different wavelengths along the sodium D lines (Fig. 20). The acquisition procedure to build the dopplergrams (that is using the two samples on the blue and red wings of the solar profile) and the true passbands of the MOF have been used to obtain the atmospheric heights corresponding to the velocity offsets (positive for reddening). We have taken into account the sensitivity of the instrument to the oscillations. In fact, the MOF passband on the red wing of the solar profile is typically more sensitive to the oscillations due to the larger slope of the solar profile itself far from the line center. This effect results in a non-balanced contribution of the opposite wings and is shown in Fig. 21, where the maximum formation height does not correspond to the line center. Note that we

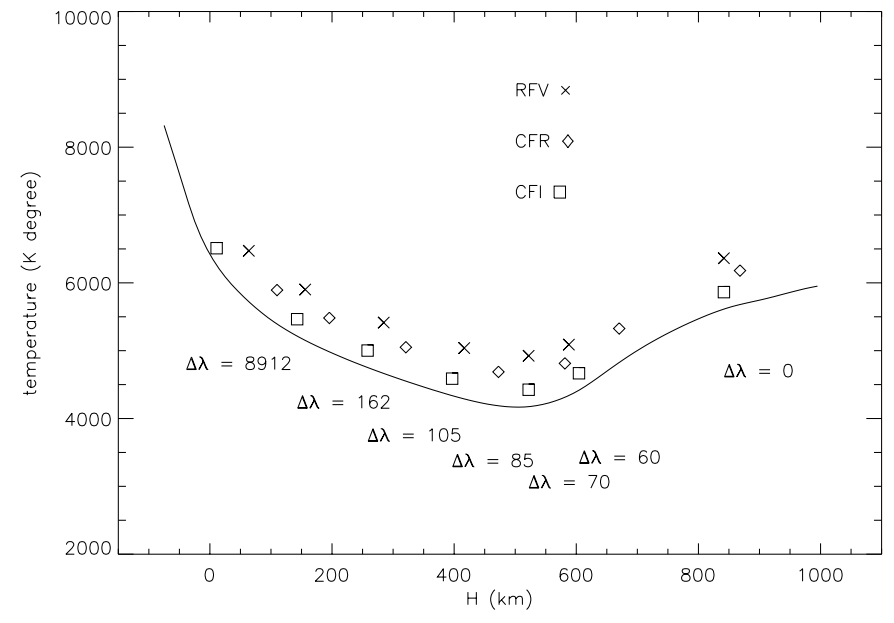

Fig. 20. The formation heights for the sodium D1 at different wavelengths from the line center (in milliAngströms). Crosses, triangles, squares refer to rensponse function for the velocity (RFV), the contribution function for the residual intensity (CFR) and line intensity (CFI) respectively. These heigths are shown at arbitrary levels overlaying a plot of the temperature vs. height from the solar VAL-C model.

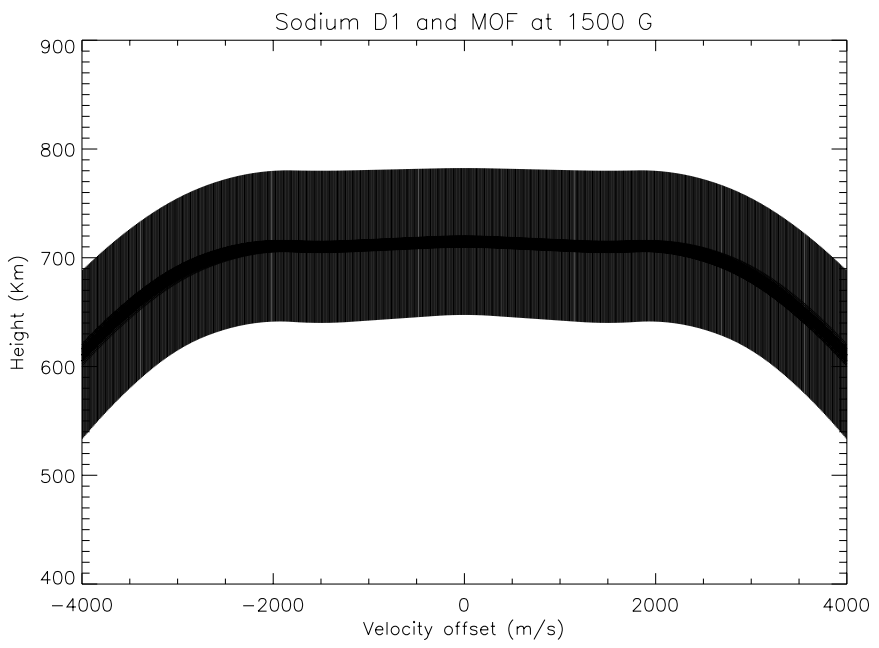

Fig. 21. The acquisition procedure used to obtain the dopplergrams with the sodium MOF has been introduced in a model in order to compute the corresponding solar heights in the solar atmosphere. The true passbands for a MOF at $1500 \mathrm{G}$ and $260{ }^{\circ} \mathrm{C}$ have been used. On the $x$-axis the velocity offset for the solar D1 is reported, instead of the wavelength, to permit an estimate of the phase values vs. height from Fig. 19. The dark area limit the heights obtained with the model. The D2 forms approximately less than $100 \mathrm{~km}$ higher (Stix 1989).

computed these heights using the rensponse function for velocity: this is not full representative of the $I-V$ phase. Nevertheless, due to the intrinsic scatter of any average formation height parameter, we believe this is useful for a comparison with the results from other authors (Deubner et al. 1990, 1996). The results from the referred observations are confirmed. 


\section{Conclusions}

The crosstalk between the intensity and velocity has been modelled for the Magneto-Optical Filters when the signals are built as the sum or the normalised difference between two intensities obtained in the opposite wings of a solar line profile. The main effect is introduced in the intensity signal by the different tuning of the filter caused by a velocity shift.

The intensity images and the dopplergrams have been analysed using a local analysis and the spherical harmonic decomposition in order to study the spatial distribution of the $I-V$ phase difference and also to produce the $I-V$ $\ell-\nu$ diagram.

Many approaches used to infer an estimate of the errors induced by this crosstalk have been shown. The most reliable procedure consists in computing the calibration curves and correct the images as they would be acquired with a filter tuned at the same wavelength along the solar line profile.

We conclude, as expected, that the modulus of the measured $I-V$ phase difference is systematically lower than the unperturbed one.

The amount of this decrease depends on the instrumental parameters that determine the transmission profiles of the filters (Figs. 3, 4 and Table 1), and the position on the disk. These parameters have to be used to compute the correction or chosen to reduce the contamination to the minimum. In our case, for a sodium MOF at $270{ }^{\circ} \mathrm{C}$ and $4^{\prime \prime} /$ pix spatial resolution, we obtain a correction of $\simeq 5^{\circ}$ for the $p$-modes in the five-minute band using only the induced intensity contribution. The correction should be roughly doubled when the induced velocity contribution is included. After the correction, we confirm, within the error bars, the values reported by Deubner et al. (1990) for the same line.

General comments on the phases obtained with narrow passband filters has to be done. First of all, a difference is shown when the signals are treated locally or with the spherical harmonics decomposition. The offset velocity makes the filter scan the solar profile across the disk. This means that, according to the height formation layers, the obtained values vary on the disk. This effect is clearly shown in the phase difference (see for example Figs. 7 and 17) and a variation during the year is expected since the relative velocity between the earth and the sun changes up to $400 \mathrm{~m} \mathrm{~s}^{-1}$.

The $\ell-\nu$ diagram performs an average over the disk and, for the $p$-modes, the phases at disk center will contribute the most.

Moreover, the phases attributed to the different phenomena strongly depend on how these are distinguished. If a threshold method is applied to select the $p$-modes, the local analysis can fail when the signal-to-noise of the modes is not high (as in the three-minute band). This effect can produce different phase values between the local and $\ell-\nu$ analysis.
The statistical error in the phase for both methods seem to be large $\left(\simeq \pm 20^{\circ}\right.$, when it is evaluated either from the phase spread over the disk, as in this paper, or from the scatter of the different $\mathrm{m}$ values, as in Oliviero et al. 1999).

In conclusion, resonance line spectrometers, like the MOFs discussed in this study, are particular susceptible to systematic errors when they infer $V$ and $I$ fluctuations from the measurement of only two points along the solar line profile (which are at different intensity levels). The aim of this paper, contributing to the quantitative understanding of these errors, is to favor a meaningful interpretation of the $I-V$ phase measurements in the solar atmosphere.

This is desirable since competitive scenarios have been proposed in recent years to explain in particular the origin of the phases in the solar background: we would like to establish, without doubt, whether the negative phase regime discovered at low frequencies by Deubner et al. (1990) is the result of waves reflected higher up in the atmosphere by the temperature stratification (Deubner et al. 1992; Marmolino et al. 1993) or whether it is the signature of those seismic events that have been claimed to excite the five-minute oscillations (Strous et al. 2000; Skartlien \& Rast 2000; Moretti et al. 2001a). Using the results from the crosstalk correction, we can suggest that the solar background is produced by a source whose ratio between the velocity and intensity fluctuations is comparable to that of the modes at our resolution.

Acknowledgements. P.F.M. thanks F. Brandizzi. We sincerely thank the referee, F. L. Deubner, for his comments. We also thank B. Cullmann, S. M. Jefferies, M. Oliviero, Th. Pettauer, Th. Straus and the MOF Development Group. The MOF Development Group is a collaboration between the Department of Physics of the University of Rome "La Sapienza", the Trieste Astronomical Observatory, the Institute of Geophysics, Astrophysics and Meteorology, the University of Graz and the Kanzelhöhe Solar Observatory. It consists of A. Cacciani, A. Hanslmeier, M. Messerotti, P. F. Moretti, W. Otruba and A. Warmuth. The data have been obtained at the Kanzelhöhe Solar Observatory.

\section{References}

Cacciani, A., Ricci, D., Rosati, P., et al. 1988, in ESA Seismology of the Sun and Sun-Like Stars, 185

Cacciani, A., \& Moretti, P. F. 1997, Sol. Phys., 175, 1

Cacciani, A., Moretti, P. F., \& Rodgers, W. E. 1997, Sol. Phys., 145,115

Cacciani, A., Moretti, P. F., Messerotti, M., et al. 1999, in Motions in the Solar Atmosphere, ed. Hanslmeier \& Messerotti (Kluwer Academic Publishers), 239, 271

Deubner, F. L., Fleck, B., Marmolino, C., \& Severino, G. 1990, A\&A, 236, 509

Deubner, F. L., Fleck, B., Schmitz, F., \& Straus, Th. 1992, A\&A, 266, 560

Deubner, F. L., Waldschik, Th., \& Steffens, S. 1996, A\&A, 307, 936

Jimenez, A., Roca Cortes, T., Severino, G., \& Marmolino, C. 1999, ApJ, 525, 1055 
Marmolino, C., Severino, G., Deubner, F. L., \& Fleck, B. 1993, A\&A, 278, 617

Marmolino, C., Oliviero, M., Severino, G., \& Smaldone, L. A. 1997, A\&AS, 125, 381

Moretti, P. F., Severino, G., Cauzzi, G., et al. 1997, in Proc. of SCORe '96: Solar Convection and Oscillations and their Relationship, ed. F. P. Pijpers, J. Christensen-Dalsgaard, \& C. Rosenthal (Kluwer Ac. Publ., Astronomy and Space Library), 225, 293

Moretti, P. F., \& The MOF Development Group, 2000, Sol. Phys., 196, 51

Moretti, P. F., Cacciani, A., Hanslmeier, A., et al. 2001a, A\&A, 372,1038

Moretti, P. F., Oliviero, M., Severino, G., \& The MOF Development Group, 2001b, in Proc. of 10th SOHO/ GONG Workshop, ESA SP-464, 661

Oliviero, M., Severino, G., \& Straus, Th. 1998, ESA SP-418, 275

Oliviero, M., Severino, G., Straus, Th., Jefferies, S. M., \& Apporchaux, T. 1999, ApJ, 516, L45
Parnell, R. L., \& Beckers, J. M. 1969, Sol. Phys., 9, 35

Rhodes, E. J., Cacciani, A., \& Tomczyk, S. 1988, in Advances in Helio and Asteroseismology, IAU Symp., 123, 471

Skartlien, R., \& Rast, M. P. 2000, ApJ, 535, 464

Severino, G., Moretti, P. F., Oliviero, M., \& The VAMOS Team 2001, in SOHO 10/ GONG 2000 Workshop: Helio- and Astero seismology at the Dawn of the Millennium, ESA SP-464, 337

Stix, M. 1989, in The Sun, ed. R. Harwit, V. Kippenhahn, J. P. Trimble, \& Zahn (Springer-Verlag Publ., Astronomy and Space Library), 143

Straus, Th., Fleck, B., Severino, G., et al. 1998, ESA SP-417, 293

Strous, L. H., Goode, P. R., \& Rimmele, T. R. 2000, ApJ, 535, 1000

Tomczyk, S., Streander, K., Card, G., et al. 1995, Sol. Phys., 159,1

Ulrich, R. K., Henney, C. J., Schimpf, S., et al. 1993, A\&A, 280,268 